\title{
Impaired Coronary Blood Flow Response to Acetylcholine in Patients with Coronary Risk Factors and Proximal Atherosclerotic Lesions
}

\author{
Kensuke Egashira, Tetsuzi Inou, Yoshitaka Hirooka, Akira Yamada, Yuji Maruoka, \\ Hisashi Kai, Masaru Sugimachi, Satoshi Suzuki, and Akira Takeshita \\ Research Institute of Angiocardiology and Cardiovascular Clinic, Faculty of Medicine, Kyushu University, Fukuoka 812, Japan
}

\begin{abstract}
We examined whether coronary risk factors and atherosclerotic lesions in the study artery were associated with impaired endothelium-dependent dilation of coronary resistance arteries. Acetylcholine (ACH) at graded doses $(1,3,10$ and $30 \mu \mathrm{g} / \mathrm{min}$ ) and papaverine $(10 \mathrm{mg})$ were selectively infused into the left anterior descending coronary artery of 28 patients, in whom the study artery was angiographically normal $(n=16)$ or with mild stenosis $\leqq 40 \%(n=12)$. Coronary blood flow (CBF) was estimated from the product of mean CBF velocity measured by an intracoronary Doppler catheter and the arterial cross-sectional area of the study artery determined by quantitative arteriography. ACH increased CBF in a dose-dependent manner. However, the maximum $\mathrm{CBF}$ response to $\mathrm{ACH}$ varied widely among patients (from $50 \%$ to $660 \%$ ). By multivariate analysis, the presence of atherosclerotic lesions in the study artery was an independent predictor for impaired CBF response to $\mathrm{ACH}(P$ $<0.01)$. Hypertension $(P<0.001)$, hypercholesterolemia $(r$ $=-0.52, P<0.005)$, age $\geqq 50$ yr $(P<0.01)$ and total number of coronary risk factors $(r=-0.62, P<0.001)$ were associated with the impaired increase in $\mathrm{CBF}$ with $\mathrm{ACH}$ by univariate analysis. The percent increase in $\mathrm{CBF}$ evoked with papaverine did not correlate with these risk factors. The results suggest that mild atherosclerotic lesions in the study artery and coronary risk factors are accompanied by impaired endothelium-dependent dilation of coronary resistance arteries evoked with ACH. Endothelial dysfunction of coronary resistance arteries may result in altered regulation of myocardial perfusion in patients with mild coronary atherosclerosis and coronary risk factors. (J. Clin. Invest. 1993. 91:29-37.) Key words: coronary circulation • endothelium-dependent vasodilation • hypercholesterolemia $\cdot$ hypertension $\bullet$ microcirculation
\end{abstract}

\section{Introduction}

It has been recognized that the vascular endothelium plays a pivotal role in regulating vascular smooth muscle tone (1-5). Studies in animals and humans demonstrated that a variety of

This study was presented in part at the Scientific Meeting of the American Heart Association, November 1991, Anaheim, CA.

Address reprint requests to Dr. Egashira, Research Institute of Angiocardiology, Kyushu University Faculty of Medicine, 3-1-1, Maidashi, Higashi-ku, Fukuoka 812, Japan.

Received for publication 8 January 1992 and in revised form 16 June 1992.

J. Clin. Invest.

(c) The American Society for Clinical Investigation, Inc. 0021-9738/93/01/29/09 \$2.00

Volume 91, January 1993, 29-37 pathologic settings such as vasospasm, hypercholesterolemia, hypertension, and atherosclerosis are associated with impaired endothelium-dependent vasorelaxation (6-13). Recent studies in humans have demonstrated that angiographically normal coronary arteries dilate and the arterial segments with atherosclerotic lesions constrict with acetylcholine $(\mathrm{ACH})^{1}$ infusion $(14,15)$. It has been shown that risk factors for coronary artery disease are associated with impaired $\mathrm{ACH}$-induced vasodilation in patients with angiographically normal coronary arteries (16). These lines of evidence imply that endothelial dysfunction of large epicardial coronary arteries develops with atherosclerosis and coronary risk factors. However, these previous studies have only studied the effect of atherosclerosis on endothelium-dependent dilation of large epicardial coronary arteries.

Recent studies have demonstrated that endothelium-dependent vasodilation occurs in resistance arteries $(17,18)$. Nitric oxide, which accounts for the biological activity of endothelium-derived relaxing factor (19), is synthesized from L-arginine (20). It has been shown that $\mathrm{L}$-arginine analogues, which inhibit synthesis of nitric oxide from L-arginine (20), decrease coronary blood flow (CBF) $(21,22)$. The latter results suggest that endothelium-dependent vasodilation of resistance artery plays an important role in determining CBF, in that Chilian et al. (23) have demonstrated that myocardial perfusion is determined predominantly by small resistance arteries $>200 \mu \mathrm{m}$ in diameter. An impaired dilation of peripheral and coronary resistance arteries in response to $\mathrm{ACH}$ has been reported in patients with hypercholesterolemia $(24,25)$. In animals with atherosclerosis in large conduit arteries, endothelium-dependent dilation of resistance coronary artery evoked with $\mathrm{ACH}$ is impaired (26-29). These findings suggest that pathophysiological manifestation of atherosclerosis may extend into the resistance artery which is free of histological evidence of atherosclerosis. To date, however, few studies have examined whether endothelial function of coronary resistance arteries is altered by the presence of proximal atherosclerotic lesions and coronary risk factors in humans. Zeiher et al. (30) demonstrated that endothelium-dependent vasodilation of resistance coronary artery evoked with $\mathrm{ACH}$ was less in normocholesterolemic patients with mild nonobstructive coronary artery disease than those without.

This study aimed to determine if risk factors for coronary artery disease and mild atherosclerotic lesions in the proximal portion of the study artery alter endothelium-dependent dilation of coronary resistance arteries in humans. For this purpose, responses of $\mathrm{CBF}$ to intracoronary infusions of $\mathrm{ACH}$ ( an

1. Abbreviations used in this paper: $\mathrm{ACH}$, acetylcholine; $\mathrm{CBF}$, coronary blood flow; CSA, cross-sectional area; LAD, left anterior descending coronary artery; LCX, left circumflex coronary artery; RCA, right coronary artery. 
endothelium-dependent vasodilator) and to an intracoronary injection of papaverine (an endothelium-independent vasodilator) were examined, and the effects of risk factors and proximal atherosclerotic lesions on $\mathrm{CBF}$ responses to drugs were examined by multiple regression analysis. The results indicated that the presence of atherosclerotic lesions in the proximal artery and coronary risk factors are associated with impaired vasodilating response of resistance coronary artery to $\mathrm{ACH}$.

\section{Methods}

Patients. The study was done in 38 patients undergoing diagnostic coronary arteriography. We examined the effects of coronary risk factors and the presence of a mild atherosclerotic lesion in the proximal left anterior descending coronary artery (LAD) on CBF response to $\mathrm{ACH}$ in 28 patients, in whom LAD (i.e., the study artery) was angiographically normal or with a mild diameter stenosis $\leqq 40 \%$. We examined the effects of ergonovine on CBF response in 5 other patients with angiographically normal coronary arteries and 5 with mild atherosclerotic lesions in the study artery. Patients with myocardial infarction, unstable angina, variant angina, valvular heart diseases, or heart failure were excluded from the study. Patients who had evidence of left ventricular hypertrophy determined by either electrocardiographic criteria or the left ventricular mass index of $>100 \mathrm{~g} / \mathrm{m}^{2}$ estimated by biplane left ventriculogram ( the lower limit of normal range in our laboratory was $<100 \mathrm{~g} / \mathrm{m}^{2}$ ) were excluded.

Coronary risk factors examined in this study included age $>50 \mathrm{yr}$, hypercholesterolemia $>220 \mathrm{mg} / \mathrm{dl}$, hypertension, cigarette smoking, and a family history of coronary artery disease. Total serum cholesterol was measured by an enzymatic method. No patients were on cholesterol lowering agents before study. Hypercholesterolemic patients who had a family history of hypercholesterolemia, thickening of Achilles tendon, or xanthoma (i.e., familial hypercholesterolemia) were excluded. Hypertension was defined as systolic and/or diastolic blood pressure $>160$ and/or $95 \mathrm{mmHg}$, respectively. Hypertensive patients were being treated with various antihypertensive drugs. Patients were considered as smokers when they had a habit of cigarette smoking or had stopped smoking within 3 mo before the study. A positive family history of coronary artery disease was thought to be present when one or more of siblings or parents had documented coronary artery disease before the age of $60 \mathrm{yr}$. Diabetes mellitus was diagnosed by the glucose tolerance test. No patients examined in this study had diabetes mellitus.

The severity of an atherosclerotic lesion was assessed by coronary angiograms recorded after an intracoronary administration of isosorbide dinitrate of $2.0 \mathrm{mg}$. 28 patients in whom the effects of $\mathrm{ACH}$ were studied were divided into two groups based on the angiographic findings of LAD (Table I); those with angiographically normal LAD without luminal irregularities or stenosis and those with luminal irregularities or diameter stenosis $\leqq 40 \%$. Patients who had diameter stenosis $>40 \%$ in LAD were excluded from the study. Of the patients with normal LAD, 10 patients had mild atherosclerotic lesions (diameter stenosis $\leqq 50 \%$ ) in the left circumflex coronary artery (LCX) and/or right coronary artery (RCA), and six patients had no luminal irregularity or stenosis in LCX and RCA. Of the patients with atherosclerotic lesions in LAD, 7 patients had mild atherosclerotic lesions in LCX or RCA, and 5 patients had a diameter stenosis of $51 \%$ to $90 \%$ in RCA and/or LCX. None of these patients with atherosclerotic lesions in LAD had diameter stenosis $>90 \%$ nor the visible collateral anastomosis on coronary angiograms. Clinical profiles of the patients are shown in Table I. 10 patients in whom the effect of ergonovine on the CBF response was studied had variable degrees of stenosis from zero to $75 \%$ in LCX or RCA.

The research protocol of this study was approved by the Institutional Committee Board for clinical research. Written informed consent was obtained from each patient.

Quantitative coronary angiography. Coronary cineangiograms were recorded using a cineangiographic system (Siemens Corp., Iselin, NJ). Nonionic contrast material (Iohexol 350, Dai-ichi Sei-Yaku Pharmaceutical Co., Tokyo, Japan) was injected by a power injector in the amount of $7 \mathrm{ml}$. An appropriate view that allowed visualization of the proximal LAD with no overlap of the other coronary arteries was selected to determine the cross-sectional area (CSA) of LAD at the segment of 2-4 mm distal to the tip of the Doppler catheter. The CSA of the proximal LAD was determined using a cinevideodensitometric analysis system (coronary analyzer, model XR-70, Vanguard Corp., Melville, NY) (31). The end-diastolic frames of single-plane angiograms were selected and projected with a Vanguard projector. The images of the LAD segment that contained the tip of the Doppler catheter were acquired into the analyzer through a video camera with twofold magnification and were digitized. The two or more fixed anatomical structures serving as references (i.e., branch points) were determined to allow assessment of serial changes in the CSA during drug infusions. The CSA of the LAD segment of 2-4 mm length was measured three times or more by a preprogrammed microprocessor system. The size of the catheter was used as a reference to calculate the absolute CSA in $\mathrm{mm}$. The mean CSA of the segment during infusion of each drug was used for later analysis. The inter- and intra-observer reproducibility of the measurements of the CSA using this system were high ( $r$ $=0.95$ and $r=0.98$, respectively).

Measurements of $C B F$ velocity and estimation of $C B F$. Diagnostic catheterization was performed by a femoral approach. A $8 \mathrm{~F}$ angioplasty-guiding catheter was introduced into the left main coronary artery. A 3F Doppler flow velocity catheter (20 MHz Mikro-Tip Doppler catheter, model DC-201, Millar Instruments, Houston, TX) was introduced into the proximal LAD. A tip of the Doppler catheter was placed at the segment without atherosclerotic lesions and in the center of the lumen. The Doppler catheter was then connected to a DC-101 Velocimeter (Millar Instruments) to obtain the mean and phasic velocity signals. Before beginning the protocol, the range gate was adjusted to obtain the high quality audio signals and phasic flow velocity waveforms. The position of the Doppler catheter and the range gate were not changed thereafter throughout the study. The use of this device to assess coronary blood flow velocity in humans has been described (32, 33). The measurements of coronary blood flow velocity were done during continuous infusion of ISDN $(40 \mu \mathrm{g} / \mathrm{min})$ to minimize changes in diameter of the proximal LAD. In preliminary studies, we examined the effects of intravenous ISDN at the low dose on CBF response to $\mathrm{ACH}(10 \mu \mathrm{g} / \mathrm{min})$ and papaverine $(10 \mathrm{mg})$ in four patients with angiographically normal coronary arteries, and found that the magnitudes of the increase in CBF evoked with these agonists did not change before and after ISDN. The percent increases in CBF evoked with ACH before and after ISDN were $373 \pm 93 \%$ and $345 \pm 61 \%$ (NS), respectively and those with papaverine before and after ISDN were $428 \pm 80 \%$ and $443 \pm 64 \%$ (NS), respectively.

$\mathrm{CBF}$ was estimated from the product of the mean CBF velocity and the CSA of the LAD segment at the tip of the Doppler catheter. The increases in estimated $\mathrm{CBF}$ in response to $\mathrm{ACH}$ and papaverine were expressed as the percent increases from the baseline value.

Study protocol. Antianginal drugs such as nitrates and calcium channel blockers were discontinued $24 \mathrm{~h}$ before the study. Cardiac catheterization was performed in the fasting state after premedication with oral diazepam of $5 \mathrm{mg}$. The study was done after completion of diagnostic catheterization.

In 28 patients with and without mild atherosclerotic lesions in the study artery (Table I), serial intracoronary administrations of drugs were performed in the following order: (a) A bolus injection of papaverine $(10 \mathrm{mg} / 5 \mathrm{ml})$ through the guiding catheter. An additional papaverine administration at a dose of $12-14 \mathrm{mg}$ was done if the percent increase in estimated CBF evoked with papaverine at a dose of $10 \mathrm{mg}$ was $<400 \%$; $(b)$ infusion of saline $(0.5 \mathrm{ml} / \mathrm{min}$ for $2 \mathrm{~min})$ through the lumen of the Doppler catheter; $(c)$ cumulative infusions of $\mathrm{ACH}(0.5$ $\mathrm{ml} / \mathrm{min}$ ) at the doses of $1,3,10$, and $30 \mu \mathrm{g} / \mathrm{min}$ (for $2 \mathrm{~min}$ at each dose) through the Doppler catheter. After completion of the study with 


\begin{tabular}{|c|c|c|c|c|c|c|c|c|c|c|}
\hline \multirow[b]{2}{*}{ No. } & \multirow[b]{2}{*}{ Age } & \multirow{2}{*}{$\begin{array}{l}\text { Gender } \\
(\mathrm{M} / \mathrm{F})\end{array}$} & \multirow{2}{*}{$\begin{array}{l}\text { Serum } \\
\text { cholesterol } \\
\text { level }\end{array}$} & \multirow[b]{2}{*}{ Hypertension } & \multirow[b]{2}{*}{ Smoking } & \multirow{2}{*}{$\begin{array}{l}\text { Family } \\
\text { history }\end{array}$} & \multirow{2}{*}{$\begin{array}{l}\text { Risk } \\
\text { Factors }\end{array}$} & \multirow{2}{*}{$\begin{array}{l}\text { Atherosclerotic lesions } \\
\text { in LAD }\end{array}$} & \multicolumn{2}{|c|}{$\begin{array}{l}\text { Maximum response } \\
\text { of CBF }\end{array}$} \\
\hline & & & & & & & & & ACH & Papaverine \\
\hline & $y r$ & & $m g / d l$ & & & & $n$ & & \multicolumn{2}{|c|}{ \% increase } \\
\hline 1 & 54 & F & 188 & - & + & - & 2 & - & 480 & 520 \\
\hline 2 & 21 & $\mathbf{F}$ & 160 & - & - & - & 0 & - & 660 & 600 \\
\hline 3 & 34 & $\mathbf{M}$ & 188 & - & + & - & 1 & - & 390 & 410 \\
\hline 4 & 43 & $\mathbf{M}$ & 230 & - & + & - & 2 & - & 460 & 580 \\
\hline 5 & 67 & $\mathrm{~F}$ & 173 & - & - & - & 1 & - & 200 & 450 \\
\hline 6 & 20 & $\mathbf{M}$ & 126 & - & + & - & 1 & - & 620 & 650 \\
\hline 7 & 61 & $\mathrm{~F}$ & 316 & - & - & - & 2 & - & 400 & 450 \\
\hline 8 & 61 & $\mathbf{M}$ & 204 & + & - & - & 2 & - & 430 & 420 \\
\hline 9 & 43 & $\mathbf{M}$ & 184 & - & + & - & 1 & - & 280 & 420 \\
\hline 10 & 53 & $\mathbf{M}$ & 190 & - & - & - & 1 & - & 620 & 420 \\
\hline 11 & 66 & $F$ & 227 & - & - & + & 3 & - & 400 & 420 \\
\hline 12 & 48 & $\mathbf{M}$ & 236 & + & + & - & 3 & - & 400 & 310 \\
\hline 13 & 59 & $F$ & 241 & + & + & + & 5 & - & 290 & 470 \\
\hline 14 & 58 & $\mathbf{M}$ & 234 & + & + & - & 4 & - & 410 & 490 \\
\hline 15 & 43 & $\mathbf{F}$ & 234 & - & - & - & 1 & - & 420 & 490 \\
\hline 16 & 38 & $\mathbf{M}$ & 203 & - & + & - & 1 & - & 610 & 640 \\
\hline 17 & 64 & $\mathrm{~F}$ & 264 & + & - & - & 3 & + & 50 & 310 \\
\hline 18 & 43 & $\mathbf{M}$ & 260 & + & + & - & 3 & + & 230 & 560 \\
\hline 19 & 73 & $\mathbf{M}$ & 188 & + & + & - & 3 & + & 80 & 310 \\
\hline 20 & 62 & $\mathbf{M}$ & 246 & + & + & - & 3 & + & 130 & 300 \\
\hline 21 & 56 & $\mathrm{~F}$ & 208 & - & - & - & 1 & + & 160 & 480 \\
\hline 22 & 73 & $F$ & 260 & + & - & + & 4 & ++ & 120 & 360 \\
\hline 23 & 73 & $\mathbf{M}$ & 241 & + & + & - & 4 & ++ & 120 & 560 \\
\hline 24 & 51 & $\mathbf{M}$ & 256 & + & + & + & 5 & + & 90 & 440 \\
\hline 25 & 55 & $\mathbf{M}$ & 279 & + & + & + & 5 & ++ & 125 & 460 \\
\hline 26 & 61 & $\mathbf{M}$ & 255 & + & + & + & 5 & ++ & 194 & 410 \\
\hline 27 & 66 & $\mathbf{M}$ & 270 & + & + & + & 5 & ++ & 220 & 590 \\
\hline 28 & 48 & $\mathbf{M}$ & 256 & + & + & + & 4 & + & 300 & 490 \\
\hline
\end{tabular}

Risk factors: number of coronary risk factors (see text). Atherosclerotic lesions in LAD: (-) no atherosclerotic lesions without luminal irregularity; $(+)$ the presence of luminal irregularity (diameter stenosis of $<25 \%$ ); $(++)$ the presence of diameter stenosis of $25-40 \%$.

one drug, we waited for at least $5 \mathrm{~min}$ before beginning infusion of the next drug, by which time the coronary diameter and CBF velocity returned to the baseline values. Each patient received all three drugs. In order to determine the maximal response, $\mathrm{ACH}$ at a dose of $60 \mu \mathrm{g} / \mathrm{min}$ was given to ten patients and confirmed that $\mathrm{ACH}$ at a dose of 60 $\mu \mathrm{g} / \mathrm{min}$ did not increase estimated CBF more than that evoked with $\mathrm{ACH}$ at a dose of $30 \mu \mathrm{g} / \mathrm{min}$. Coronary arteriography was performed before and $1 \mathrm{~min}$ after papaverine and $2 \mathrm{~min}$ after saline or $\mathrm{ACH}$. In particular, after infusion of $\mathrm{ACH}$ at the dose of $30 \mu \mathrm{g} / \mathrm{min}$, coronary angiograms in several projections were taken to exclude the development of a flow-limiting constriction of the epicardial LAD.

In five other patients with normal LAD and in five with mild atherosclerotic lesions in LAD, ergonovine at a dose of $10 \mu \mathrm{g}$ was infused by bolus into the left main coronary artery through the guiding catheter. Coronary arteriography was performed before and 2 min after ergonovine. This study was done to examine whether mild constriction of epicardial LAD by ergonovine affected baseline CBF and hyperemic response of $\mathrm{CBF}$ to the contrast medium (iohexol $7 \mathrm{ml}$ ). The same amount of iohexol was injected before and after ergonovine through the guiding catheter which was positioned at the same place.

The mean and phasic CBF velocity, arterial pressure, heart rate, and standard 12-lead electrocardiograms were continuously monitored and recorded on a multi-channel recorder (polygraph system, Nihon
Kohden, Tokyo). Values during a steady-state condition were used for later analysis.

Statistical analysis. Data are expressed as mean \pm SD. We examined the effects of risk factors for coronary artery disease and mild atherosclerotic lesions in the proximal portion of the study artery on the maximum $\mathrm{CBF}$ response to $\mathrm{ACH}$ and $\mathrm{CBF}$ response to papaverine. Simple linear regression analysis was used to examine the effects of continuous variables (the serum cholesterol level, baseline hemodynamic variables, and the baseline CSA) on the maximum CBF response to drugs. The total number of coronary risk factors (among the following factors: the total cholesterol level $>220 \mathrm{mg} / \mathrm{dl}$, age $\geq 50 \mathrm{yr}$, a positive family history of coronary artery disease, hypertension, and cigarette smoking) was considered as a continuous variable and thus, examined by linear regression analysis. The unpaired Student's $t$ test was used to examine effects of coronary risk factors (hypertension, age $>50 \mathrm{yr}$, smoking, family history, and gender) and proximal atherosclerotic lesions on the $\mathrm{CBF}$ response evoked with $\mathrm{ACH}$ or papaverine. Finally, the effects of various risk characteristics on the $\mathrm{CBF}$ responses to $\mathrm{ACH}$ and papaverine were examined by the multiple stepwise linear regression analysis. When serial changes in hemodynamic variables such as arterial pressure, heart rate, and the arterial CSA were compared between patients with and without proximal atherosclerotic lesions, analysis of variance (ANOVA) for repeated measures followed by Bon- 
Table II. Averaged Changes in Hemodynamic Variables during the Study

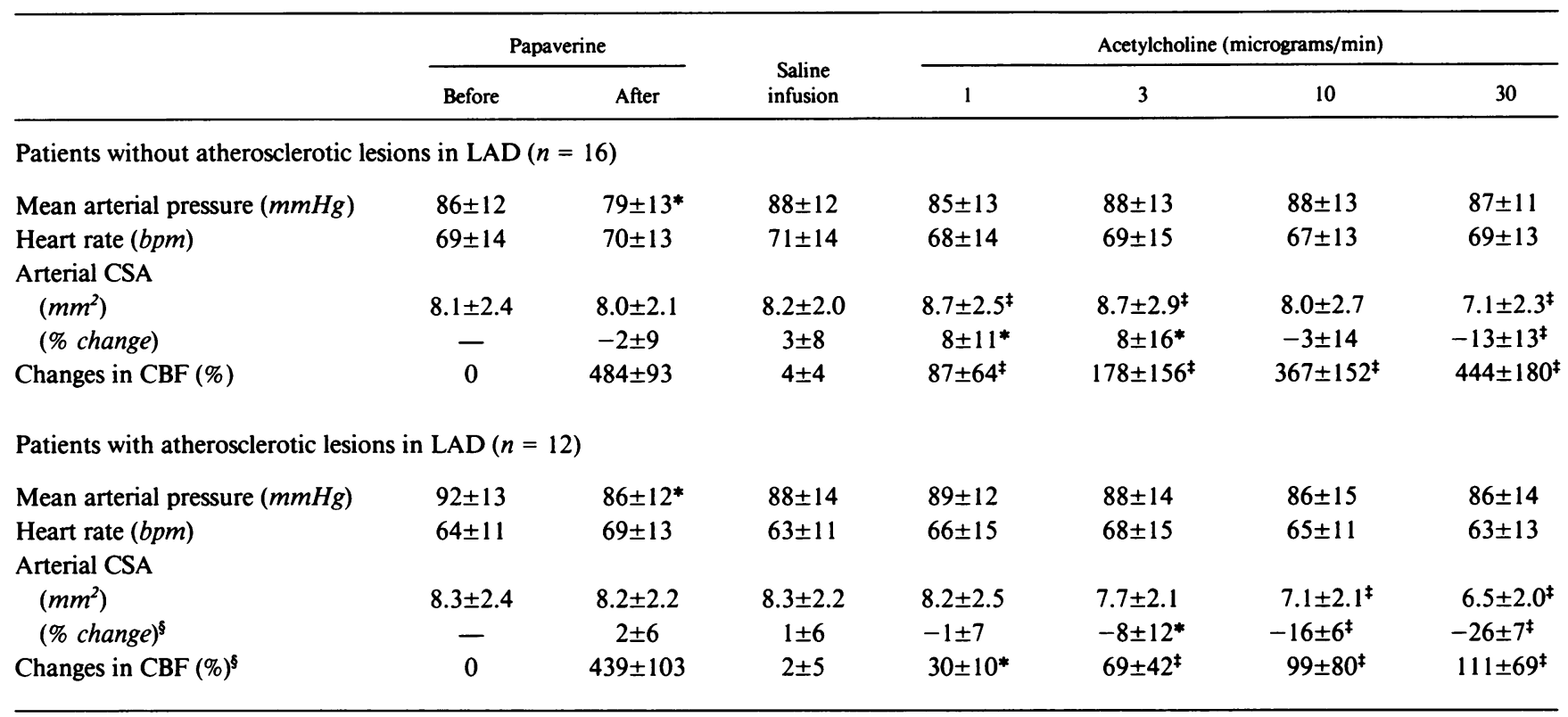

Data are mean \pm SD. Averaged values of patients with and without atherosclerotic lesions in LAD (the study artery) are presented. Estimated CBF are shown as the percent increases from the value before papaverine. ${ }^{*} P<0.05 ;{ }^{\ddagger} P<0.01$ vs. before papaverine (ANOVA plus Bonferroni's test). ${ }^{\S}$ There are significant differences in the percent changes arterial CSA and percent changes in CBF evoked with $\mathrm{ACH}$ at the graded doses between patients with and without atherosclerotic lesions in $\operatorname{LAD}(P<0.01$ by ANOVA).

ferroni's multiple comparison test was used (Table II). The unpaired $t$ test was used to examine the effect of atherosclerotic lesions and coronary risk factors on the large epicardial coronary artery response to $\mathrm{ACH}$ ( Table III). The probability level $<0.05$ was considered as significant.

\section{Results}

Hemodynamic response. Intracoronary infusion of $\mathrm{ACH}$ did not alter mean arterial pressure and heart rate (Table II). Infusion of papaverine did not significantly change heart rate, but slightly decreased mean arterial pressure (Table II).

Table III. Effects of Atherosclerotic Lesions and Coronary Risk Factors on the Large Epicardial Response to $\mathrm{ACH}$ at the Dose That Caused the Maximal Increase in CBF

\begin{tabular}{llll}
\hline & \multicolumn{3}{c}{$\begin{array}{c}\text { Percent decrease in CSA } \\
\text { of proximal LAD }\end{array}$} \\
\cline { 2 - 4 } \multicolumn{1}{c}{ Factor } & + & - & $P$ value \\
\hline $\begin{array}{l}\text { Percent decrease in CSA in the large epicardial coronary } \\
\quad \text { evoked with ACH }\end{array}$ & & & \\
Atherosclerotic lesions in proximal & & & \\
$\quad$ LAD & $23 \pm 8$ & $12 \pm 8$ & 0.002 \\
Hypercholesterolemia & $20 \pm 7$ & $13 \pm 12$ & 0.04 \\
Hypertension & $22 \pm 7$ & $10 \pm 7$ & 0.0001 \\
Age $\geqq 50$ yr & $20 \pm 8$ & $11 \pm 9$ & 0.02 \\
Family history of coronary artery & & & \\
$\quad$ disease & $21 \pm 7$ & $15 \pm 10$ & NS \\
Smoking & $19 \pm 10$ & $13 \pm 8$ & NS \\
Male gender & $19 \pm 10$ & $14 \pm 7$ & NS \\
& & & \\
\hline
\end{tabular}

Representative recordings of $\mathrm{CBF}$ velocity during $\mathrm{ACH}$ infusions in a patient are presented in Fig. 1, which denotes the dose-dependent increases in $\mathrm{CBF}$ velocity evoked with $\mathrm{ACH}$ with no changes in mean arterial pressure and heart rate.

Effects of coronary risk factors and proximal atherosclerotic lesions on large epicardial coronary artery response to $A C H$. $\mathrm{ACH}$ at the doses of 1 and $3 \mu \mathrm{g} / \mathrm{min}$ increased the CSA of LAD and $\mathrm{ACH}$ at the doses of 10 and $30 \mu \mathrm{g} / \mathrm{min}$ progressively decreased the CSA in patients without atherosclerotic lesions in LAD (Table II). In contrast, in patients with atherosclerotic lesions in $\mathrm{LAD}, \mathrm{ACH}$ at graded doses consistently and progressively decreased the CSA (Table II). The percent decreases in the epicardial CSA with graded doses of ACH were significantly greater in patients with atherosclerotic lesions in LAD than in those without $(P<0.01$ by ANOVA). The CSA did not significantly change with papaverine.

The effects of atherosclerotic lesions and various coronary risk factors on the percent decrease in the epicardial CSA evoked with $\mathrm{ACH}$ at the dose that produced the maximum CBF response are presented in Table III. The percent decrease in the epicardial CSA evoked with $\mathrm{ACH}$ was greater in patients with atherosclerotic lesions, hypercholesterolemia, hypertension, and age $\geqq 50$ yr than those without, and was not different between patients with and without risk factors such as smoking, a family history of coronary artery disease, and male gender.

Effects of coronary risk factors and proximal atherosclerotic lesions on $C B F$ response to $A C H$. The percent maximum increase in CBF evoked with $\mathrm{ACH}$ and with papaverine in each patient are shown in Table $\mathrm{I}$. The maximum increase in CBF with $\mathrm{ACH}$ varied widely from $50 \%$ to $660 \%$ among patients (Table I). The averaged percent increases in CBF in patients with and without atherosclerotic lesions in LAD are shown in Table II. Saline infusion did not alter CBF. ACH at graded 


\section{SALINE ACETYLCHOLINE}

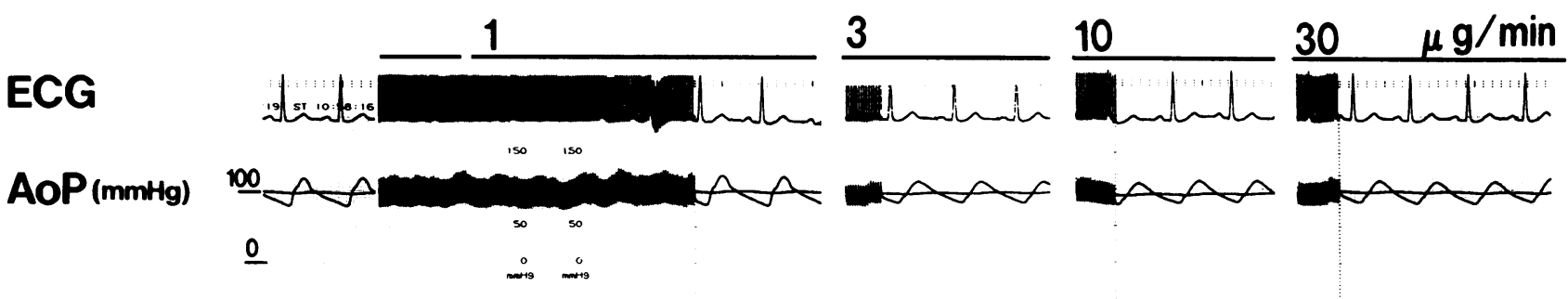

\section{$\operatorname{CBFV(cm/s)}$}

phasic
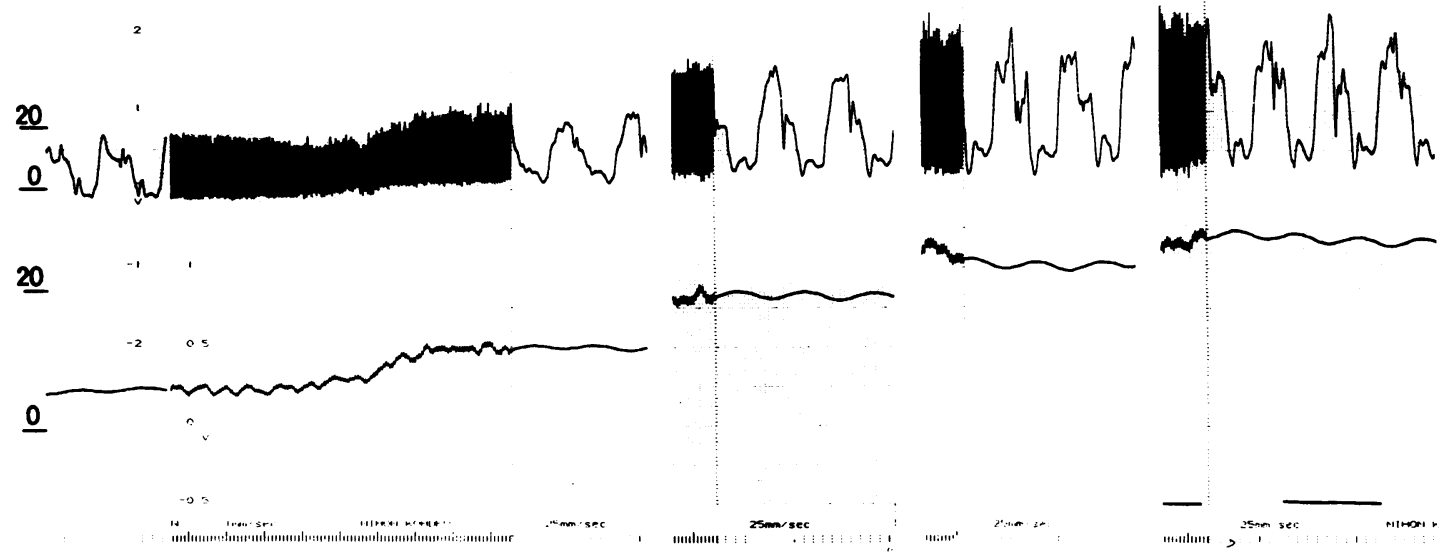

$10 \mathrm{~s} 1 \mathrm{~s}$

Figure 1. Representative recordings of phasic and mean CBF velocity ( $C B F V)$, systemic arterial pressure ( $A o P)$, and electrocardiogram ( $E C G$, II lead) during $\mathrm{ACH}$ infusion in a patient. Intracoronary $\mathrm{ACH}$ at the graded doses increases $\mathrm{CBFV}$ in a dose-dependent fashion with no changes in mean arterial pressure and heart rate.

doses resulted in the dose-dependent increases in CBF in the two groups, but the progressive increases in $\mathrm{CBF}$ in patients with atherosclerotic lesions in LAD were less than those in patients without atherosclerotic lesions in $\operatorname{LAD}(P<0.01$ by ANOVA). The percent increases in CBF with papaverine did not differ between patients with and without atherosclerotic lesions in LAD. The percent increases in CBF with papaverine in the two groups were comparable to those reported previously in patients without significant coronary artery disease $(18,19)$.

The percent maximum increase in $\mathrm{CBF}$ evoked with $\mathrm{ACH}$ did not correlate with baseline hemodynamic variables such as heart rate, mean systemic arterial pressure, cardiac output, left ventricular filling pressure, and the baseline CSA of LAD (data not shown). The percent increase in CBF evoked with papaverine also did not correlate with these variables (data not shown).

The percent maximum increase in $\mathrm{CBF}$ with $\mathrm{ACH}$ in patients with proximal atherosclerotic lesions in LAD $(152 \pm 72 \%)$ was significantly less $(P<0.001)$ than that in patients without atherosclerotic lesions $(442 \pm 131 \%)$ (Fig. 2). The percent maximum increase in $\mathrm{CBF}$ in the two groups shown in Fig. 2 is not the same as the percent increase in CBF evoked with $\mathrm{ACH}$ at $30 \mu \mathrm{g} / \mathrm{min}$ shown in Table II, since the percent maximum increase in $\mathrm{CBF}$ was not necessarily evoked with the highest dose of $\mathrm{ACH}$. The percent increase in $\mathrm{CBF}$ with papaverine did not differ between patients with and without atherosclerotic lesions in LAD $(P=0.24)$ (Table II, Fig. 2).

The percent maximum increase in CBF evoked with $\mathrm{ACH}$ correlated negatively with the serum cholesterol level $(r$
$=-0.52, P<0.005$ ) (Fig. 3). In contrast, no significant correlation was found between the percent increase in CBF evoked with papaverine and the serum cholesterol level. The percent maximum increase in $\mathrm{CBF}$ with $\mathrm{ACH}$ was significantly less $(P$ $<0.001)$ in hypertensive $(213 \pm 127 \%)$ than in normotensive patients $(439 \pm 161 \%$ ) (Fig. 4). The percent increase in CBF evoked with papaverine in hypertensive patients $(432 \pm 98 \%)$ tended to be less than that in normotensive patients $(502 \pm 87 \%)(P=0.057)$. However, the ratio of the maximum CBF response evoked with $\mathrm{ACH}$ to the $\mathrm{CBF}$ response evoked with papaverine was smaller $(P=0.004)$ in hypertensive patients $(0.50 \pm 0.32)$ than in normotensive patients $(0.87 \pm 0.29)$

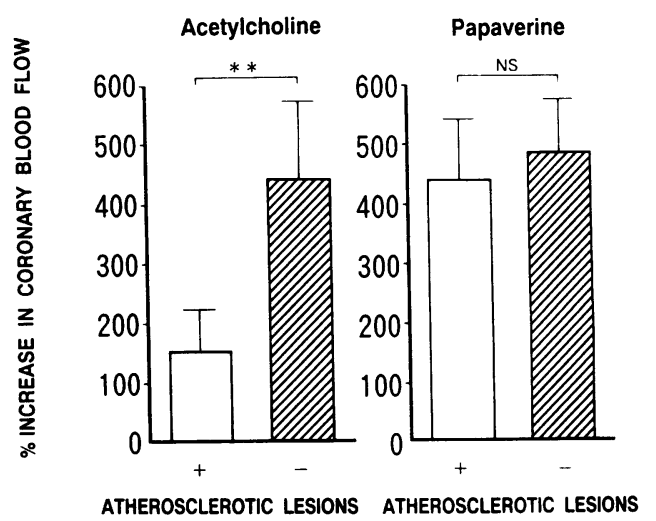

Figure 2. Summary of the percent increases in $\mathrm{CBF}$ evoked with $\mathrm{ACH}$ and papaverine in patients with and without atherosclerotic lesions in the study artery. ${ }^{* *} P<0.001$ by unpaired $t$ test. 


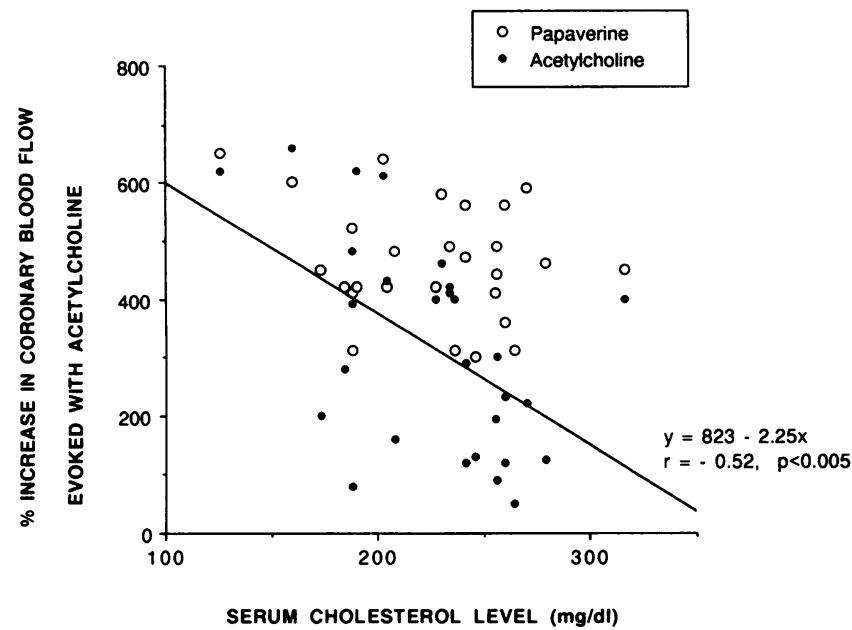

Figure 3. Scatter plots of the relation between the percent increase in $\mathrm{CBF}$ evoked with $\mathrm{ACH}$ and the serum cholesterol level (•). By simple linear regression analysis, there is a significant negative correlation between the percent increase in $\mathrm{CBF}$ evoked with $\mathrm{ACH}$ and the serum cholesterol level. However, no significant correlation is found between the percent increase in CBF evoked with papaverine and the serum cholesterol level (O).

(Fig. 4). Age greater than 50 years was associated with the impaired increase in $\mathrm{CBF}$ evoked with $\mathrm{ACH}(P<0.01)$ as well as with papaverine $(P<0.05)$ (Fig. 5). However, the ratio of the maximum $\mathrm{CBF}$ response evoked with $\mathrm{ACH}$ to the $\mathrm{CBF}$ response evoked with papaverine was smaller $(P<0.05)$ in patients with age $\geqq 50 \mathrm{yr}$ than in those with age $<50$ years (Fig. $5)$. Total number of coronary risk factors also correlated with the impaired maximum $\mathrm{CBF}$ response to $\mathrm{ACH}(r=-0.61, P$ $<0.001$ ), whereas there was no significant correlation between total number of coronary risk factors and the $\mathrm{CBF}$ response to papaverine (Fig. 6). There was no significant difference in the

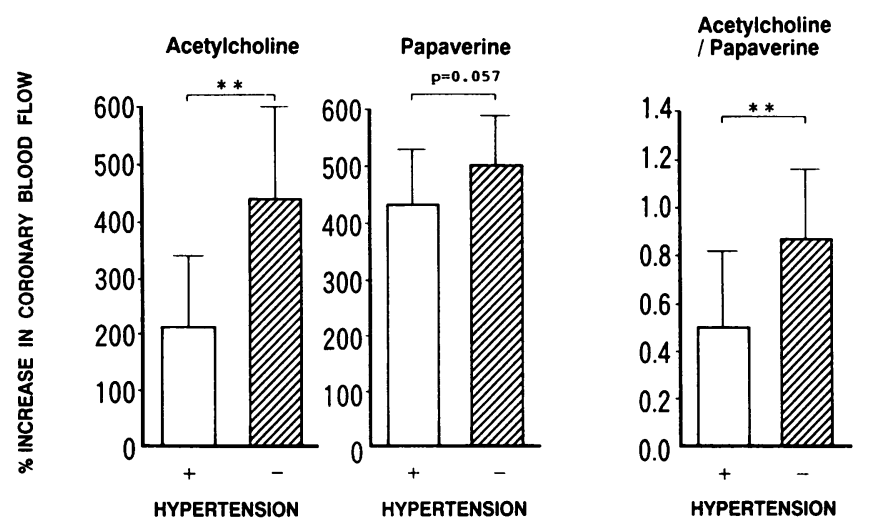

Figure 4. Comparison of the percent increases in CBF evoked with $\mathrm{ACH}$ and papaverine between hypertensive and normotensive patients. Hypertensive patients have a significantly attenuated CBF response to $\mathrm{ACH}$ as compared to normotensive patients. Although the difference in the $\mathrm{CBF}$ response to papaverine between the two groups approaches a significant difference $(P=0.057)$, the ratio of the percent maximum increase in $\mathrm{CBF}$ evoked with $\mathrm{ACH}$ to the percent increase in CBF evoked with papaverine is significantly $(P<0.01)$ smaller in hypertensive patients than in normotensive patients. ${ }^{* *} P$ $<0.01$ by unpaired $t$ test.

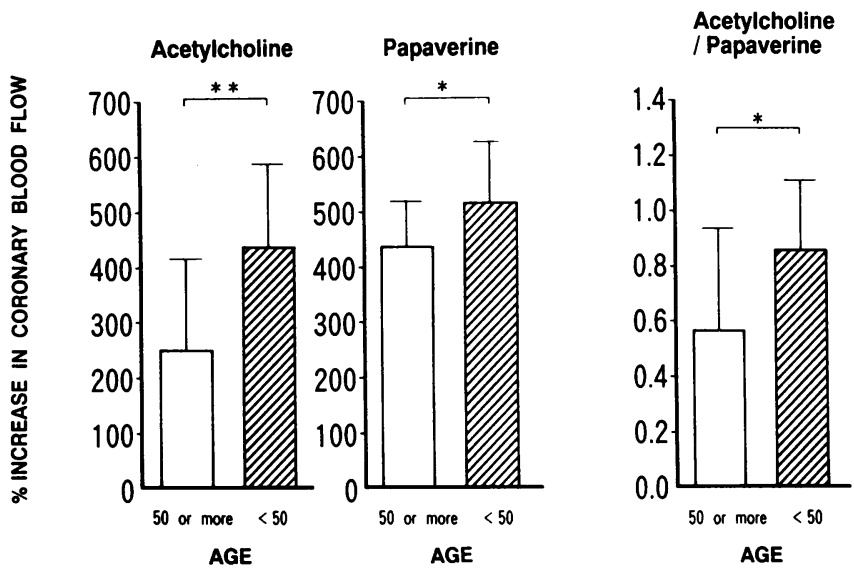

Figure 5. Comparison of the $\mathrm{CBF}$ response to $\mathrm{ACH}$ and papaverine between patients with their age $\geq 50 \mathrm{yr}$ and patients with their age $<50 \mathrm{yr}$. Age $\geq 50 \mathrm{yr}$ was associated with the impaired increase in CBF evoked with ACH $(P<0.01)$ as well as with papaverine $(P$ $<0.05)$. However, the ratio of the maximum $C B F$ response evoked with $\mathrm{ACH}$ to the $\mathrm{CBF}$ response evoked with papaverine was signifcantly $(P<0.05)$ smaller in patients with age $\geqq 50 \mathrm{yr}$ than in those with age $<50$ yr. ${ }^{*} P<0.05 ;{ }^{* *} P<0.01$ by unpaired $t$ test.

maximum $\mathrm{CBF}$ response to $\mathrm{ACH}$ between patients with and without cigarette smoking or a family history of coronary artery disease (data not shown). Male gender also did not alter the maximum CBF response to $\mathrm{ACH}$ (data are not shown).

Multiple linear regression analysis revealed that the maximum $\mathrm{CBF}$ response evoked with $\mathrm{ACH}$ negatively correlated with the presence of atherosclerotic lesions in the proximal LAD $(P<0.01)$. The correlations between the CBF response evoked with $\mathrm{ACH}$ and other factors such as the serum cholesterol level, hypertension, age $\geqq 50 \mathrm{yr}$, and total number of coronary risk factors were not significant by multiple regression analysis.

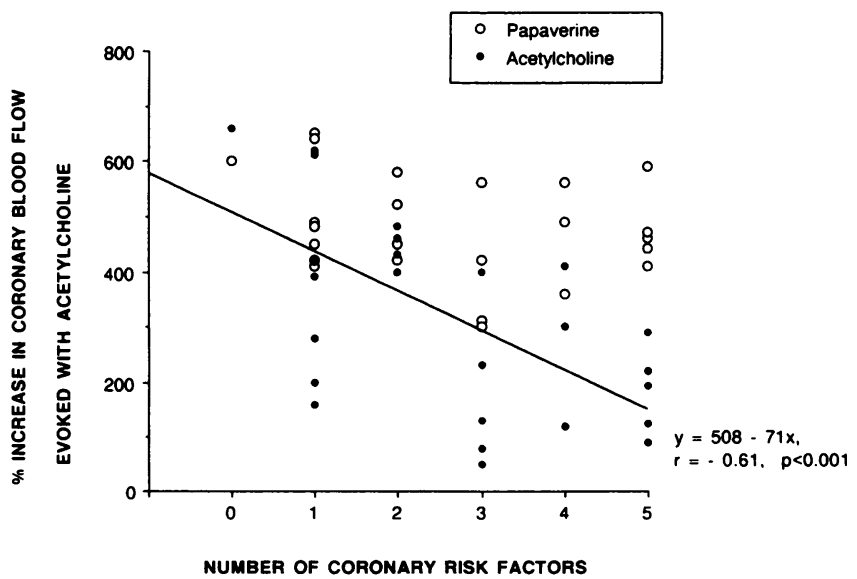

Figure 6. Scatter plots of the relation between the CBF response with $\mathrm{ACH}$ and number of coronary risk factors existed in the patients $(\bullet)$. By simple linear regression, there is a significant negative correlation between the $\mathrm{CBF}$ response with $\mathrm{ACH}$ and number of coronary risk factors. However, no significant correlation is found between the CBF response with papaverine and number of coronary risk factors $(O)$. 
Effects of ergonovine-induced constriction of the large epicardial coronary artery and $C B F$. An injection of ergonovine $(10 \mu \mathrm{g})$ into the left main coronary artery decreased the CSA in LAD by $24 \pm 10 \%$ in patients with normal LAD and $30 \pm 9 \%$ in patients with atherosclerotic lesion in LAD (NS vs. patients with normal LAD), which were comparable with maximum vasoconstriction induced by $\mathrm{ACH}$ in patients with and without atherosclerotic lesions in LAD. Ergonovine did not changed baseline CBF. The hyperemic increases in CBF velocity evoked with the contrast medium were comparable before and after ergonovine ( $196 \pm 37$ and $230 \pm 40 \%$ [NS] in patients with normal LAD, and $224 \pm 41$ and $240 \pm 45 \%$ [NS] in patients with atherosclerotic lesions in $\mathrm{LAD}$, respectively). We did not assess the changes in CBF evoked with the contrast medium, because changes in arterial CSA were not assess by repeating arteriography before and immediately after the injection of contrast medium. However, it is assumed that changes in CBF velocity evoked with the contrast medium seem to be nearly identical with those in $\mathrm{CBF}$, because the contrast medium per se may have little effect on the CSA.

\section{Discussion}

The major finding of this study is that the presence of mild atherosclerotic lesions in the study coronary artery was associated, by multiple regression analysis, with the attenuated increase in estimated CBF evoked with intracoronary $\mathrm{ACH}$. In addition, hypertension, hypercholesterolemia, age $\geqq 50 \mathrm{yr}$, and total number of coronary risk factors also correlated with the impaired increase in $\mathrm{CBF}$ evoked with $\mathrm{ACH}$ by univariate analysis. In contrast, the increase in CBF with papaverine was not significantly altered by the presence of mild atherosclerotic lesions, hypertension, hypercholesterolemia, or total number of coronary risk factors. These results suggest that the presence of mild atherosclerotic lesions in the study artery and some coronary risk factors impair endothelium-dependent vasodilation of coronary resistance arteries. In Discussion that follows, we will consider the validity of the methods employed in the study and the new findings in light of previous observations.

We made several assumptions in the methods to assess the magnitude of endothelium-dependent vasodilation of coronary resistance arteries. First, it was assumed that $\mathrm{ACH}$ dilated coronary resistance arteries by means of the endothelium-dependent mechanism. This assumption appears reasonable, because it has been shown that $\mathrm{ACH}$-induced dilation of resistance arteries was prevented by hemoglobin, methylene blue, and a L-arginine analogue (26-29). In humans, the increase in forearm blood flow evoked with $\mathrm{ACH}$ was inhibited by $\mathrm{L}-\mathrm{N}^{\mathrm{G}}$ monomethyl-arginine (18), which inhibits the conversion from L-arginine to nitric oxide in endothelial cells. Hodgson and Marshall (34) showed in humans that the increases in CBF evoked with $\mathrm{ACH}$ were inhibited after pretreatment with methylene blue.

Second, it was assumed that the increases in CBF evoked with drugs reflected vasodilation of coronary resistance arteries and were not limited by organic or dynamic stenosis in the proximal large epicardial coronary artery. In fact, the presence of mild atherosclerotic lesion in the study artery did not significantly limit CBF response to papaverine (Table II). However, we should consider the possibility that dynamic stenosis of the proximal LAD evoked with ACH might have limited the in- crease in $\mathrm{CBF}$ with $\mathrm{ACH}$. As shown previously (14-16, 34), we confirmed that $\mathrm{ACH}$ at the high doses constricted the large epicardial coronary artery, and the degrees of vasoconstriction evoked with $\mathrm{ACH}$ at the high doses were greater in patients with proximal atherosclerotic lesions than in those without ( Table III); the presence of risk factors such as hypercholesterolemia, hypertension, and age $\geqq 50 \mathrm{yr}$ was associated with a greater decrease in the epicardial CSA evoked with $\mathrm{ACH}$. However, the percent reduction in the CSA evoked with $\mathrm{ACH}$ was small so that presumably it would not attenuate CBF (35). We showed that comparable vasoconstriction of proximal artery evoked with ergonovine did not affect the increase in CBF evoked with the contrast medium. Tatineni et al. (36) demonstrated that diffuse narrowing of LAD with a $12 \pm 5 \%$ decrease in the diameter (comparable to the decrease in CSA by ACH in our patients with atherosclerotic lesions in LAD), that was caused by an intravenous administration of ergonovine ( 0.4 $\mathrm{mg}$ ), did not attenuate the maximal hyperemic CBF response with papaverine $(10 \mathrm{mg})$. Thus, we consider it unlikely that limited CBF response to $\mathrm{ACH}$ in patients with atherosclerotic lesions in proximal LAD resulted from mild vasoconstriction of proximal LAD induced by $\mathrm{ACH}$.

Third, we also assumed that the continuous infusion of ISDN at the low dose did not modify CBF response to $\mathrm{ACH}$ and papaverine, in that it is demonstrated that nitrate compounds at low doses dilate preferentially large epicardial coronary arteries and have little effect on resistance arteries (32-34, $37,38)$. In fact, we found in the preliminary studies that ISDN at the low dose used in this study did not alter the magnitude of the percent increase in $\mathrm{CBF}$ in response to $\mathrm{ACH}$ or papaverine.

Fourth, we estimated CBF from the product of mean CBF velocity and the CSA at the tip of the Doppler catheter. The CSA was carefully assessed by use of a cinevideodensitometric analysis system. CBF velocity was recorded by use of an intracoronary Doppler catheter $(32,33)$. It has been shown that relative changes in CBF velocity recorded by the intracoronary Doppler catheter correlate highly with changes in CBF velocity measured by an epicardial suction Doppler probe and with changes in coronary sinus blood flow assessed by time-volume collections (32). Furthermore, in this study, the results with papaverine served as internal control for those with $\mathrm{ACH}$, since each patient received both $\mathrm{ACH}$ and papaverine infusion. We analyzed the results with $\mathrm{ACH}$ in reference to those with papaverine.

An important finding in this study is that the presence of atherosclerotic lesions in the proximal portion of the study artery was associated with impaired $\mathrm{CBF}$ response to $\mathrm{ACH}$ by multiple regression analysis. The percent increase in $\mathrm{CBF}$ with papaverine did not differ with and without proximal atherosclerotic lesions. As we previously discussed, we consider it unlikely that impaired CBF response to $\mathrm{ACH}$ in patients with proximal atherosclerotic lesions resulted from mild vasoconstriction in proximal artery induced by $\mathrm{ACH}$. These results suggest that endothelium-dependent dilation of resistance coronary arteries evoked with $\mathrm{ACH}$ was impaired in the presence of mild upstream atherosclerotic lesions. Our results in humans seem to be the foremost confirmation of animal studies by Harrison et al. (28) and Chilian et al. (29), who demonstrated impaired endothelium-dependent relaxation of resistance coronary arteries in atherosclerotic monkeys with histologic evidence of atherosclerosis in the proximal coronary artery, indi- 
cating that the pathophysiology of atherosclerosis may extends into the coronary microcirculation. Our results are compatible with the findings of a recent study by Zeiher et al. (30), who demonstrated that vasodilator capacity of resistance coronary artery to $\mathrm{ACH}$ was less in normocholesterolemic patients with mild coronary artery disease than those without. However, in the study by Zeiher et al. (30), the mean ages significantly differed between the two groups, which might have contributed to the difference in the endothelium-dependent dilation of the coronary microvasculature between the two groups. Nevertheless, the present study and the study by Zeiher et al. (30) have provided evidence suggesting that the presence of proximal atherosclerosis is associated with altered endothelial function of resistance coronary artery in humans, a unique finding implying that functional states of resistance coronary artery are affected by the presence of proximal atherosclerosis.

The precise mechanisms underlying the impaired increase in $\mathrm{CBF}$ evoked with $\mathrm{ACH}$ in the presence of proximal atherosclerotic lesions cannot be deduced from our study. However, there are several mechanisms that may possibly explain this finding. First, it is possible that patients with mild atherosclerotic lesions in large epicardial coronary arteries might have diffuse atherosclerotic changes in epicardial as well as resistance coronary arteries compared to patients with angiographically normal coronary arteries. Atherosclerotic change in resistance artery might be associated with the reduced release of endothelium-derived relaxing factors or greater constriction of vascular smooth muscle in response to $\mathrm{ACH}$ so that increase in CBF evoked with ACH were limited. Indeed, experimental studies have indicated that mild atherosclerosis impairs endothelium-dependent dilation as well as augments constricting response of vascular smooth muscle to vasoactive substances (6-16). Second, it is possible that the resistance coronary arteries might have been exposed to the increased concentrations of vasoconstricting substances such as endothelium-derived, platelet- and/or macrophage-derived vasoconstricting factors which might be activated at the upstream atherosclerotic lesions (39). These vasoactive substances might have impaired endothelial function of peripheral coronary arteries. Further studies are needed to clarify the mechanism underlying endothelial dysfunction of resistance coronary artery in the presence of proximal atherosclerotic lesions.

Other interesting findings of this study are that coronary risk factors including hypercholesterolemia, hypertension, age $\geqq 50 \mathrm{yr}$ and total number of risk factors were associated with attenuated $\mathrm{CBF}$ response to $\mathrm{ACH}$ by univariate analysis. The percent increase in CBF with papaverine was not altered by hypercholesterolemia or total number of coronary risk factors. There was a trend of impaired CBF response with papaverine in patients with hypertension and of age $\geqq 50 \mathrm{yr}$. However, the ratio of $\mathrm{CBF}$ response with $\mathrm{ACH}$ to that with papaverine was lower in patients with hypertension and of age $\geqq 50$ yr than those without hypertension and of age $<50 \mathrm{yr}$, respectively. These results suggest that these coronary risk factors were associated with endothelial dysfunction of coronary resistance arteries. Our results are consistent with the previous findings, in which hypercholesterolemia and hypertension are associated with impaired endothelium-dependent vasodilation of resistance arteries in animals and humans in coronary and peripheral vascular beds (24-29, 40-42). Vita et al. (16) have recently shown in humans that coronary risk factors (hypercho- lesterolemia, male gender, family history of coronary artery disease, and age) were associated with loss of endothelium-dependent vasodilation of large epicardial coronary artery evoked with $\mathrm{ACH}$. Thus, the results of this study and those of Vita et al. (16) suggest that coronary risk factors alter endothelial function of epicardial as well as resistance coronary arteries in humans. However, this study included patients with and without mild atherosclerotic lesions in the study artery, and the patients without atherosclerotic lesions in the study artery had atherosclerotic lesions in other coronary branches. In order to better define the association between the $\mathrm{CBF}$ response to $\mathrm{ACH}$ and the coronary risk factors, further studies are needed in the patients who have angiographically normal coronary arteries.

The present study may have important clinical implications, because endothelium-derived relaxing factors plays an pivotal role in myocardial perfusion (21-23). Indeed, basal coronary flow and reactive hyperemia was attenuated in isolated hearts after inhibition of nitric oxide synthesis with a L-arginine analogue (43). Kuo et al. (44) suggested that impaired endothelium-dependent vasodilating responses of coronary microvessels isolated from atherosclerotic pigs were restored after administration of L-arginine. A recent clinical study by Nabel et al. (45) suggested that increases in heart rate and myocardial oxygen consumption by rapid atrial pacing were associated with the attenuated increase in CBF in patients who had mild atherosclerotic lesions in the proximal epicardial coronary artery, compared with control patients who had angiographically normal coronary arteries. In conclusion, our results suggest that in humans endothelium-dependent vasodilation of coronary resistance arteries evoked with $\mathrm{ACH}$ is impaired with the presence of angiographical evidence of early atherosclerosis in the proximal study artery and coronary risk factors such as hypertension, aging, and hypercholesterolemia. Endothelial dysfunction of coronary resistance arteries may result in altered regulation of myocardial perfusion and thus contribute to the pathogenesis of myocardial ischemia in patients with mild coronary atherosclerosis and coronary risk factors.

\section{Acknowledgments}

This study was supported in part by grants from Uehara Memorial Foundation, Tokyo, by the Society of Clinical Pharmacology, Tokyo, Japan, by Grants-in-Aid for Scientific Research 02404045 and 02454259, and by Grant-in-Aid for Scientific Research on Priority Areas 03268226 from the Ministry of Education, Science and Culture, Tokyo, Japan.

\section{References}

1. Furchgott, R. F., and J. V. Zawadzki. 1980. The obligatory role of endothelial cells in the relaxation of arterial smooth muscle by acetylcholine. Nature (Lond.). 299:273-276.

2. Vanhoutte, P. M. 1988. The endothelium: modulation of vascular smooth muscle tone. N. Engl. J. Med. 319:512-513.

3. Bassenge, E., and R. Busse. 1988. Endothelial modulation of coronary tone. Prog. Cardiovasc. Dis. 30:349-380.

4. Lücher, T. F., V. Richard, M. Tschudi, Z. Yang, and C. Boulanger. 1990. Endothelial control of vascular tone in large and small coronary arteries. J. Am. Coll. Cardiol. 15:519-527.

5. Myers, P. R., P. F. Banit, R. Guerra, Jr., and D. G. Harrison. 1989. Characteristics of canine resistance arteries: importance of endothelium. Am. J. Physiol. 257:H603-610.

6. Vanhoutte, P. M., and D. S. Houston. 1985. Platelets, endothelium and vasospasm. Circulation. 72:728-734. 
7. Yamamoto, Y., H. Tomoike, K. Egashira, and M. Nakamura. 1987. Attenuation of endothelium-related relaxation and enhanced responsiveness of vascular smooth muscle to histamine in spastic coronary arterial segments from miniature pigs. Circ. Res. 61:772-778.

8. Tomoike, H., K. Egashira, Y. Yamamoto, and M. Nakamura. 1989. Enhanced responsiveness of smooth muscle, impaired endothelium-dependent relaxation and genesis of coronary spasm. Am. J. Cardiol. 63:33E-39E.

9. Habib, J. B., C. Bossaler, S. Wells, C. Williams, J. D. Morrisett, and P. D. Henry. 1986. Preservation of endothelium-dependent relaxation in cholesterolfed rabbit by treatment with the calcium blocker PN 200110. Circ. Res. 58:305309.

10. Freiman, P. C., G. C. Mitchell, D. D. Heistad, M. L. Armstrong, and D. G. Harrison. 1986. Atherosclerosis impairs endothelium-dependent vascular relaxation to acetylcholine and thrombin in primates. Circ. Res. 58:783-789.

11. Verbeuren, T. J., F. H. Jordaens, L. L. Zonnekeyn, C. E. Van Hove, M. C. Coene, and A. G. Herman. 1986. Effects of hypercholesterolemia on vascular reactivity in the rabbit. I. Endothelium-dependent and endothelium-independent contractions and relaxations in isolated arteries of control and hypercholesterolemic rabbits. Circ. Res. 58:552-564.

12. Lücher, T. F., and P. M. Vanhoutte. Endothelium-dependent contractions to acetylcholine in the aorta of spontaneously hypertensive rats. Hypertension. 8:344-348.

13. Bossaler, C., G. B. Habib, H. Yamamoto, C. Williams, S. Wells, and P. D. Henry. 1987. Impaired muscarinic endothelium-dependent relaxation and cyclic guanosine 5'-monophosphate formation in atherosclerotic human coronary artery and rabbit aorta. J. Clin. Invest. 79:170-174.

14. Ludmer, P. L., A. P. Selwyn, T. L. Shock, R. R. Wayne, G. H. Mudge, R. W. Alexander, and P. Ganz. 1986. Paradoxical vasoconstriction induced by acetylcholine in atherosclerotic coronary arteries. N. Engl. J. Med. 315:10461051.

15. Werns, S. W., J. A. Walton, H. H. Hsia, E. G. Nabel, M. L. Sanz, and B. Pitt. 1989. Evidence of endothelial dysfunction in angiographically normal coronary arteries of patients with coronary artery disease. Circulation. 79:287-291.

16. Vita, J. A., C. B. Treasure, E. G. Nabel, J. M. McLenachan, R. D. Fish, A. C. Yeung, V. I. Vekshtein, A. P. Selwyn, and P. Ganz. 1990. Coronary vasomotor response to acetylcholine relates to risk factors for coronary artery disease. Circulation. 81:491-497.

17. Griffith, T. M., D. H. Edwards, R. L. I. Dadies, T. J. Harrison, and K. T. Evans. 1987. EDRF coordinates the behaviour of vascular resistance vessels. Nature (Lond.). 329:442-445.

18. Vallance, P., J. Collier, and S. Moncada. 1989. Effects of endothelium-dependent nitric oxide on peripheral arteriolar tone in man. Lancet. 2:997-1000.

19. Palmer, R. M. J., A. G. Ferrige, and S. Moncada. 1987. Nitric oxide release accounts for the biological activity of endothelium-derived relaxing factor. Nature (Lond.). 327:524-526.

20. Palmer, R. M. J., D. S. Ashton, and S. Moncada. 1988. Vascular endothelial cells synthesize nitric oxide from L-arginine. Nature (Lond.). 333:664-666.

21. Kelm, M., and J. Schrader. 1990. Control of coronary vascular tone by nitric oxide. Circ. Res. 66:1561-1575.

22. Kuo, L., J. Davis, and W. M. Chilian. 1990. Endothelium-dependent, flow-induced dilation of isolated coronary arterioles. Am. J. Physiol. 259(Heart Circ. Physiol.):H1063-H1070.

23. Chilian, W. M., C. L. Eastham, and M. L. Marcus. 1986. Microvascular distribution of coronary vascular resistance in beating left ventricle. Am. J. Physiol. 251 (Heart Circ. Physiol.):H779-H786.

24. Creager, M. A., J. P. Cooke, M. E. Mendelsohn, S. J. Gallagher, S. M. Coleman, J. Loscalzo, and V. J. Dzau. 1990. Hypercholesterolemia attenuates endothelium-mediated vasodilation in man. J. Clin. Invest. 86:228-234.

25. Zeiher, A. M., H. Drexler, H. Wollschlyger, and H. Just. 1991. Modulation of coronary vascular tone in humans: progressive endothelial dysfunction with different early stages of coronary atherosclerosis. Circulation. 83:391-401.
26. Yamamoto, H., C. Bossaller, J. Cartwright, and P. D. Henry. 1988. Videomicroscopic demonstration of defective cholinergic arteriolar vasodilation in atherosclerotic rabbit. J. Clin. Invest. 81:1752-1758.

27. Osborne, J. A., M. J. Siegman, A. W. Sedar, S. U. Mooers, and A. M. Lefer. 1989. Lack of endothelium-dependent relaxation in coronary resistance arteries of atherosclerotic rabbit. Am. J. Physiol. 256:C591-597.

28. Sellke, F. W., M. L. Armstrong, and D. G. Harrison. 1990. Endotheliumdependent vascular relaxation is abnormal in the coronary microcirculation of atherosclerotic primates. Circulation. 81:1586-1593.

29. Chilian, W. M., K. C. Dellsperger, S. M. Layne, C. L. Easthman, M. A. Armstrong, M. L. Marcus, and D. D. Heistad. 1990. Effects of atherosclerosis on the coronary microcirculation. Am. J. Physiol. 258(Heart Circ. Physiol.):H529H539.

30. Zeiher, A. M., H. Drexler, H. Wollschläger, and H. Just. 1991. Endothelial dysfunction of the coronary microvasculature is associated with impaired coronary blood flow regulation in patients with early atherosclerosis. Circulation. 84:1984-1992.

31. Nichols, A. B., C. F. O. Gabrieli, J. J. Fenoglio, and P. D. Esser. 1984. Quantification of relative coronary arterial stenosis by videodensitometric analysis of the coronary angiograms. Circulation. 69:512-522.

32. White, C. W., R. F. Wilson, and M. L. Marcus. 1988. Methods of measuring myocardial blood flow in humans. Prog. Cardiovasc. Dis. 31:79-94.

33. Wilson, R. F., and C. W. White. 1986. Intracoronary papaverine; an ideal coronary vasodilator for studies of coronary circulation in conscious humans. Circulation. 73:444-451.

34. Hodgson, J. M., and J. J. Marshall. 1989. Direct vasoconstriction and endothelium-dependent vasodilation: mechanisms of effects on coronary flow and arterial diameter in patients with nonstenotic coronary arteries. Circulation. 79:1043-1051.

35. Gould, K. L., and K. Lipscomb. 1974. Effects of coronary stenosis on coronary flow reserve and resistance. Am. J. Cardiol. 34:48-55.

36. Tatineni, S., M. J. Kern, and F. Aguirre. 1992. The effect of ergonovine on coronary vasodilatory reserve in patients with angiographically normal coronary arteries. Am. Heart. J. 123:617-620.

37. Vrolix, M. C., J. Piessens, and H. D. Geest. 1991. Diameter and blood flow velocity changes in nonstenotic human coronary arteries after intracoronary injection of SIN-1. J. Cardiovasc. Pharmacol. 17 (Suppl. 3):S293-299.

38. Armstrong, P. W., J. A. Armstrong, and G. S. Marks. 1980. Pharmacokinetics of intravenous nitroglycerin in congestive heart failure. Circulation. 62:160.

39. Rubanyi, G. M., R. L. Frye, D. R. Holmes, Jr., and P. M. Vanhoutte. 1987. Vasoconstrictor activity of coronary sinus plasma from patients with coronary artery disease. J. Am. Coll. Cardiol. 9:1243-1249.

40. Andrews, H. E., K. R. Bruckdorfer, R. C. Dunn, and M. Jacobs. 1987. Low-density lipoproteins inhibit endothelium-dependent relaxation in rabbit aorta. Nature (Lond.). 327:237-239.

41. Kugiyama, K., S. A. Kerns, J. D. Morrisett, R. Roberts, and P. D. Henry. 1989. Impairment of endothelium-dependent arterial relaxation by lysolecithin in modified low-density lipoproteins. Nature (Lond.). 344:160-162.

42. Linder, L., W. Kiowski, F. R. Buhler, and T. F. Lücher. 1990. Indirect evidence for release of endothelium-derived relaxing factor in human forearm circulation in vivo. Blunted response in essential hypertension. Circulation. 81:1762-1767.

43. Kostic, M., and J. Schrider. 1992. Role of nitric oxide in reactive hyperemia of the guinea pig heart. Circ. Res. 70:208-212.

44. Kuo, L., M. J. Davis, S. Cannon, and W. M. Chilian. 1992. Pathophysiological consequences of atherosclerosis extend into the coronary microcirculation. Restoration of endothelium-dependent responses by L-arginine. Circ. Res. 70:465-476.

45. Nabel, N. G., A. P. Selwyn, and P. Ganz. 1990. Paradoxical narrowing of atherosclerotic coronary arteries induced by increases in heart rate. Circulation. 81:850-859. 